\title{
Abnormalities in Inflammatory Cytokines Confer Susceptible to Chronic Neuropathic Pain-related Anhedonia in a Rat Model of Spared Nerve Injury
}

\author{
Xi Fang ${ }^{1}$, Gaofeng Zhan ${ }^{1}$, Jie Zhang ${ }^{1}$, Hui $\mathrm{Xu}^{1}$, Bin Zhu', Yimin $\mathrm{Hu}^{3}$, Chun Yang ${ }^{1}$, Ailin Luo ${ }^{1}$ \\ ${ }^{1}$ Department of Anesthesiology, Tongji Hospital, Tongji Medical College, Huazhong University of Science and Technology, Wuhan, ${ }^{2}$ Department \\ of Critical Care Medicine, The Third Affiliated Hospital of Soochow University, Changzhou, ${ }^{3}$ Department of Anestesiology, The Second Affiliated \\ Hospital of Nanjing Medical University, Nanjing, China
}

\begin{abstract}
Objective: Patients with chronic neuropathic pain (CNP) have a higher incidence to develop depression. However, its pathogenesis has not yet been fully elucidated. Here we aimed to investigate the role of inflammatory cytokines in CNP-related anhedonia, which is a core symptom of depression, and to explore the effects of ketamine and parecoxib on pain and anhedonia.

Methods: A rat model of spared nerve injury (SNI) was constructed to mimic CNP. Hierarchical cluster analysis of sucrose preference test (SPT) was applied to classify the SNI rats into anhedonia susceptible and unsusceptible. Inflammatory cytokines in medial prefrontal cortex (mPFC) of brain, serum and L2-5 spinal cord were measured. Moreover, effects of ketamine or parecoxib on mechanical withdrawal test (MWT) and SPT in anhedonia susceptible rats were detected.

Results: Tumor necrosis factor (TNF)- $\alpha$ was increased in mPFC, serum and and spinal cord of anhedonia susceptible rats. Furthermore, anhedonia susceptible and unsusceptible rats both increased the interleukin (IL)-1 $\beta$ level in mPFC, serum and spinal cord. IL-6 was altered in serum and spinal cord, but not in mPFC. IL-10 was significantly altered in $\mathrm{mPFC}$ and serum, but not in spinal cord. Additionally, ketamine treatment significantly attenuated the decreased results of MWT and SPT in anhedonia susceptible rats, and that parecoxib significantly improved the MWT score, but failed to alter the result of SPT.

Conclusion: These findings suggest that abnormalities in inflammatory cytokines confer susceptible to anhedonia in a rat model of SNI. Ketamine, a fast-acting antidepressant, has pharmacological benefits to alleviate pain and anhedonia symptoms.
\end{abstract}

KEY WORDS: Anhedonia; Pain; Neurogenic inflammation; Ketamine.

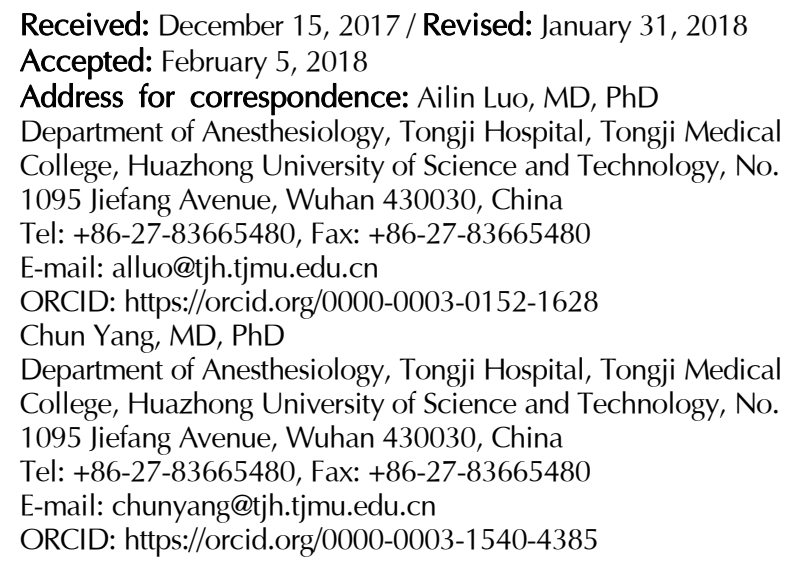

Received: December 15, 2017 / Revised: January 31, 2018

Accepted: February 5, 2018

Address for correspondence: Ailin Luo, MD, PhD

Department of Anesthesiology, Tongji Hospital, Tongji Medical

College, Huazhong University of Science and Technology, No.

1095 Jiefang Avenue, Wuhan 430030, China

Tel: +86-27-83665480, Fax: +86-27-83665480

E-mail: alluo@tjh.tjmu.edu.cn

ORCID: https://orcid.org/0000-0003-0152-1628

Chun Yang, MD, PhD

Department of Anesthesiology, Tongji Hospital, Tongji Medical

College, Huazhong University of Science and Technology, No.

1095 Jiefang Avenue, Wuhan 430030, China

Tel: +86-27-83665480, Fax: +86-27-83665480

E-mail: chunyang@tjh.tjmu.edu.cn

ORCID: https://orcid.org/0000-0003-1540-4385

\section{INTRODUCTION}

Patients with chronic neuropathic pain (CNP) are often accompanied by depression. ${ }^{1,2)}$ A previous study indicates that the average incidence of depression in patients with chronic pain is up to $52 \%{ }^{3)}$ Clinically, analgesics, e.g., nonsteroidal anti-inflammatory drugs and opioid drugs, are routinely prescribed for alleviation of CNP symptoms. ${ }^{4,5)}$ As to refractory $\mathrm{CNP}$, it has been widely recognized that antidepressant agents improving CNP-induced dysfunctional monoamine including 5-hydroxytryptamine and norepinephrine are also conventionally required. ${ }^{6,7)}$ In addition to antidepressants, analgesic agents, however, are also effective in relieving the

(ㄷ) This is an Open-Access article distributed under the terms of the Creative Commons Attribution Non-Commercial License (http://creativecommons.org/licenses/by-nc/4.0) which permits unrestricted non-commercial use, distribution, and reproduction in any medium, provided the original work is properly cited. 
symptoms of depression, particularly depression with somatic symptoms. ${ }^{8,9)}$ We therefore inferred that there are an amazing number of commonalities in the pathological mechanisms between pain and depression.

Anhedonia has emerged as one of the most core symptom of depression. ${ }^{10,11)}$ It is clinically characterized by the loss of pleasure or deficit of physiological reactivity to pleasurable stimuli. ${ }^{12)}$ Sucrose preference test (SPT) has been commonly applied to evaluate the symptoms of depression and the therapeutic effects of antidepressant agents in rodents. ${ }^{13,14)}$ Studies from our group and others suggest that depression-like phenotype rodents exert a remarkable decrease in the sucrose preference. ${ }^{15-17)}$ In contrast, improvement of poor performance in the SPT would promise a better outcome of pharmacological efficacy of antidepressant agents. ${ }^{15,18,19)}$ Currently, patients with anhedonia have a higher incidence to commit suicide. ${ }^{10)}$ It is therefore noteworthy that earlier identification of anhedonia is an urgent need to take strategies for preventing suicide in patients with depression.

Increasing evidence shows that dysfunctional inflammatory response might play an important role, at least partially, in the onset of depression. ${ }^{20,21)} \mathrm{A}$ meta-analysis study demonstrates that the expressions of inflammatory cytokines in peripheral serum of depressed patients are significantly higher than that of healthy controls. ${ }^{22)}$ In contrast, pharmacological strategies inhibiting over-activation of the inflammatory response greatly contribute to elicit beneficial effects on depression symptoms. ${ }^{23,24)}$ It is reported that intracerebroventricular micro-injection of interleukin (IL)-1 $\beta$ antagonist could exert facilitating effects on depression. ${ }^{25)}$ Similarly, tumor necrosis factor (TNF)- $\alpha$ inhibitors have currently been applied for treatment of rheumatoid arthritis, ${ }^{26)}$ several lines of evidence, however, show that inflammation inhibitors are also effective in the treatment of central nervous system diseases, e.g., depression, anxiety, Alzheimer's disease. ${ }^{27,28)}$ Furthermore, CNP-induced depressive-like phenotype is highly associated with TNF-dependent hippocampal plasticity. ${ }^{29)}$ In addition to pharmacological strategies, other therapeutic strategies, e.g., electroshock and transcranial magnetic stimulation, could also exert inhibitory effects on the serum levels of pro-inflammatory cytokines in patients with depression. ${ }^{30,31)}$ However, there are no reports on the relationship between the levels of inflammatory cytokines in various regions and CNP-elicited anhedonia susceptible in a rodent model.

In the present study, we selected several regions consisting of medial prefrontal cortex (mPFC) of brain, serum and intumescentia lumbalis of spinal cord to determine the role of inflammatory response in CNP-induced anhedonia susceptible in a rat model of SNI because alterations in inflammatory cytokines in these selected regions are considered to contribute to the pathogeneses of depression and CNP. Furthermore, ketamine or parecoxib was administrated into anhedonia susceptible rats to observe their effects on pain and anhedonia symptoms in a rat model of CNP.

\section{METHODS}

\section{Animals}

Male Sprague Dawley rats (weighing 180-230 g) were purchased from the Laboratory Animal Centre of Tongji Medical College, Huazhong University of Science and Technology (Wuhan, China). The animals were housed under 12 hour light/dark cycle with free access to food and water. Procedures of this animal experiment were in accordance with the National Institute of Health Guide for the Care and Use of Laboratory Animals. The experimental protocols were approved by the Experimental Animal Committee of Tongji Hospital, Tongji Medical College, Huazhong University of Science and Technology (No. 11400700295537).

\section{Experimental Design}

As depicted in Figure 1A, rats were acclimated to environment for 6 days then the MWT was performed 1 day before the $\mathrm{SNI}$ surgery for basal measurements. The MWT, SPT were implemented from days 5 to 8,12 to 15 and 19 to 22 after surgery, respectively. Twenty-three days after SNI surgery, mPFC of brain, serum and L2-5 spinal cord were collected for measurement of inflammatory cytokines. Additionally, in Figure 2A, anhedonia susceptible rats were intraperitoneally injected with either ketamine (Cat No.: 1707032; Gutian Pharmaceutical Company, Ningde, China) or parecoxib (Cat No.: S37009; Pfizer Pharmaceuticals Co. Ltd., New York, NY, USA) at a single dose of $10 \mathrm{mg} / \mathrm{kg}$. The SPT and MWT were performed respectively. 
A

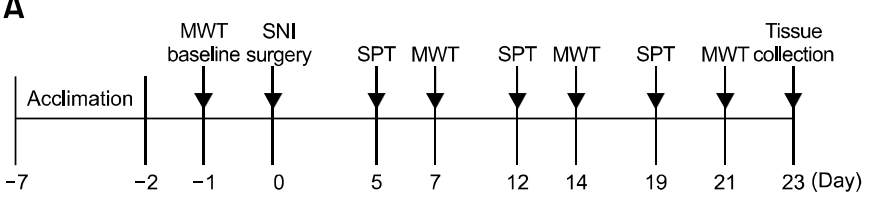

B

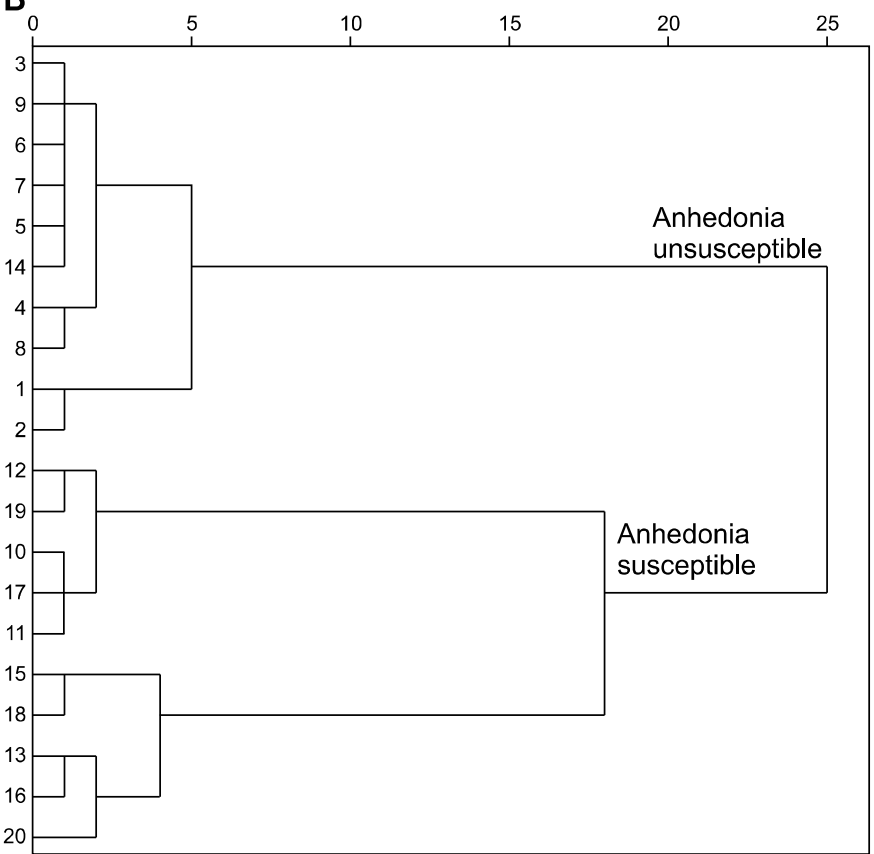

C
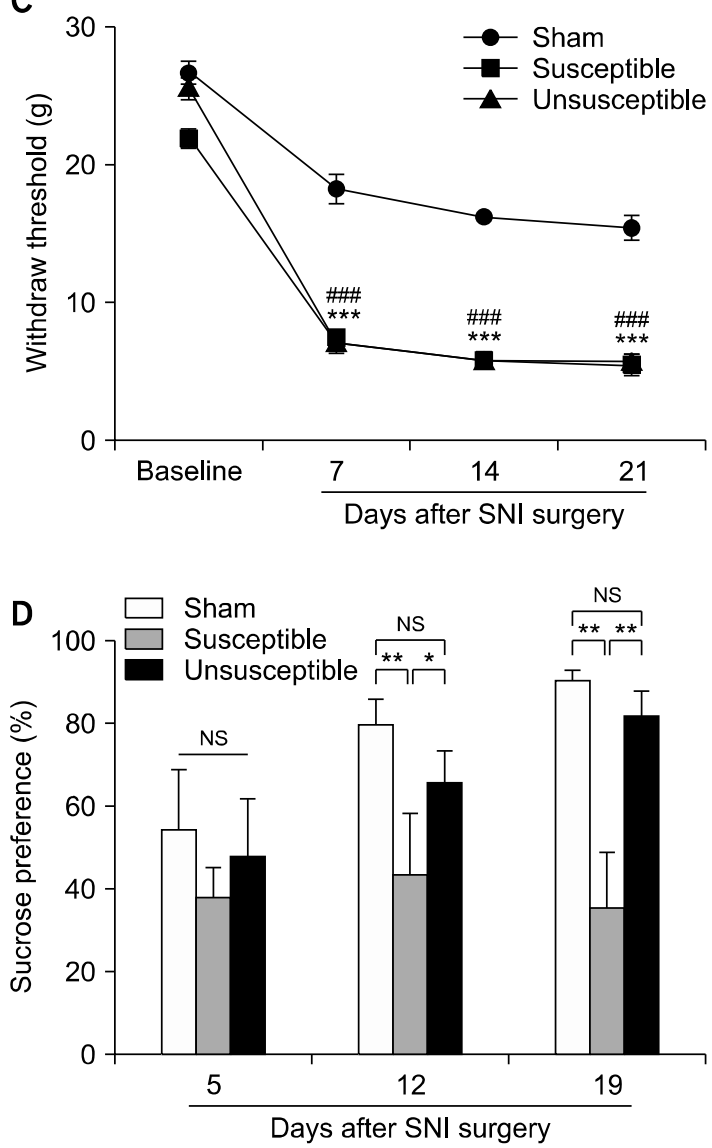

Fig. 1. Comparisons of mechanical withdrawal test (MWT) and sucrose preference test (SPT) in sham, anhedonia susceptible and unsusceptible rats. (A) The schedule of spared nerve injury (SNI) modeling and behavioral tests. SNI was performed 7 days after acclimation. Withdraw threshold was measured on day 7, 14 and 21 after SNI modeling, respectively. SPT was performed on day 5, 12 and 19 after SNI modeling, respectively. (B) Dendrogram of hierarchical clustering analysis. SNI rats were divided into anhedonia susceptible and unsusceptible groups by SPT results of hierarchical clustering analysis. (C) The results of $M W T$ (time: $\mathrm{F}_{3,18}=659.864, p<0.001$; group: $\mathrm{F}_{2,12}=96.951, p<0.001$; interaction: $\mathrm{F}_{6,36}=18.013$, $p<0.001$ ) in the sham, anhedonia susceptible and unsusceptible groups. ${ }^{* * *} p<0.001$, susceptible vs. sham group; ${ }^{\# \# \# ~} p<0.001$, unsusceptible vs. sham group. (D) SPT (time: $\mathrm{F}_{2,12}=3.125, p=0.081$; group: $\mathrm{F}_{2,12}=4.558, p=0.034$; interaction: $\mathrm{F}_{4,24}=3.178, p=0.031$ ) were measured. ${ }^{*} p<0.05$ or ** $p<$ 0.01 . Data are shown as mean \pm standard error of mean $(n=7-10)$.

NS, not significant.

\section{Spared Nerve Injury (SNI)}

The SNI surgery was performed as previously described ${ }^{32)}$ Rats were anesthetized with $10 \%$ chloral hydrate $(3 \mathrm{ml} / \mathrm{kg}$ ) and then the skin of left thigh was incised. The sciatic nerve and its three terminal branches after bluntly dissecting biceps femoris muscle were totally exposed. The common peroneal and tibial nerves were ligated with a 4-0 silk and cut off the distal to the ligation. The muscle and skin were sutured with a 4-0 silk. Rats in the Sham group were exposured to the sciatic nerve and its three terminal branches but without ligated and cut off the common peroneal and tibial nerves.

\section{Mechanical Withdrawal Test (MWT)}

Before MWT, rats were placed in plexiglass chambers with a wire net floor for 30 minutes avoiding the stress resulting from the test conditions. The Electronic Von Frey (Ugo Basile SRL, Milan, Italy) filaments were applied to the lateral $1 / 3$ of right paws. The paws quick withdrawal or flinching was considered as a positive response. Every filament stimuli were applied 4 times with a period of 30-second interval. ${ }^{33)}$

\section{Sucrose Preference Test (SPT)}

Rats were exposed to water and $1 \%$ sucrose solution for 48 hours, followed by 24 hours of water and food depri- 

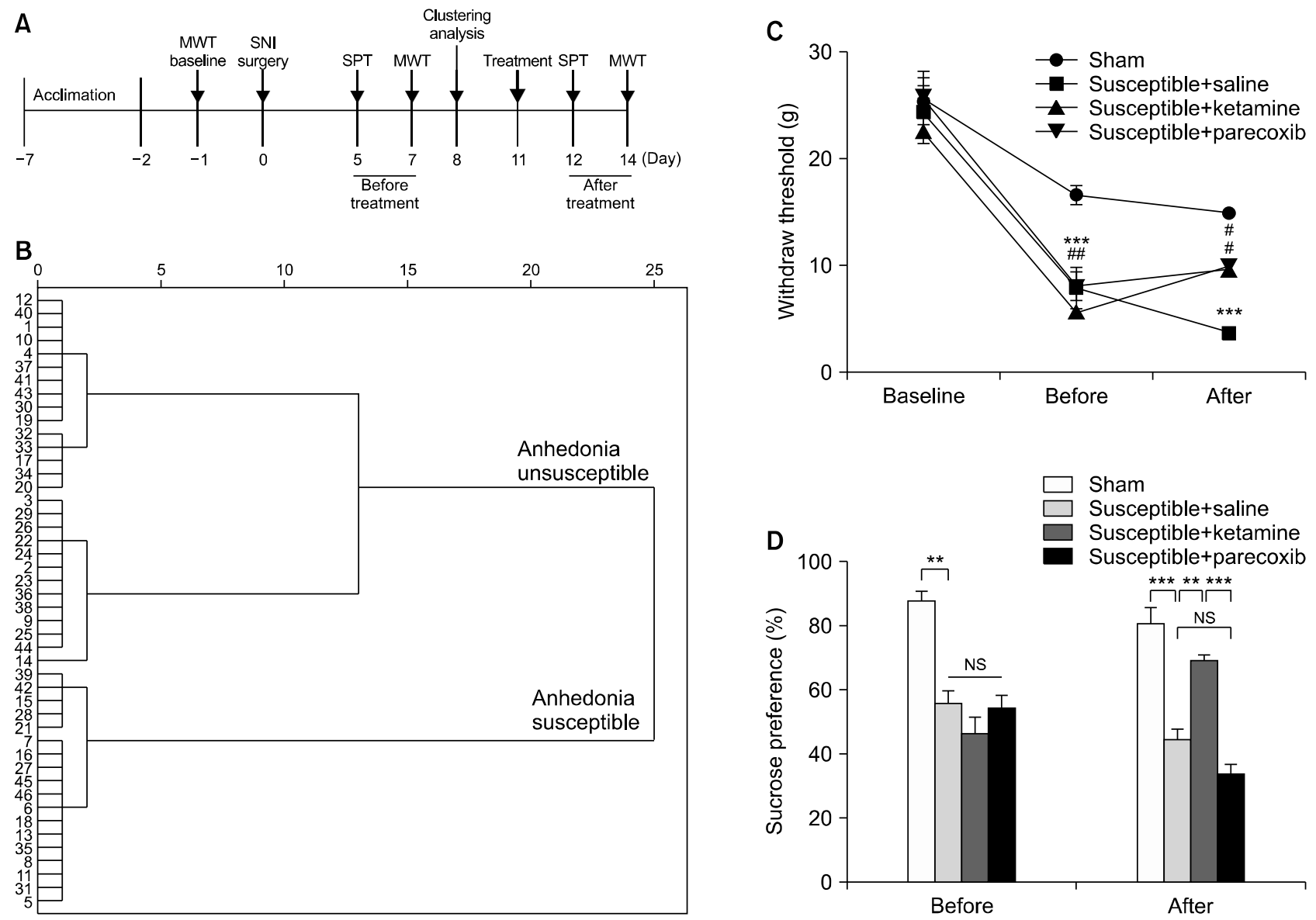

Fig. 2. Effects of ketamine and parecoxib on mechanical withdrawal test (MWT) and sucrose preference test (SPT). (A) The schedule of spared nerve injury (SNI) modeling and behavioral tests. SNI was performed 7 days after acclimation. MWT was measured on day 7 and 14 after SNI modeling, respectively. SPT was performed on day 5 and 12 after SNI modeling, respectively. Ketamine or parecoxib was intraperitoneally injected at a dose of $10 \mathrm{mg} / \mathrm{kg}$ on day 8. (B) Dendrogram of hierarchical clustering analysis. SNI rats were divided into anhedonia susceptible and unsusceptible groups by SPT results of hierarchical clustering analysis. (C) MWT (time: $F_{2,10}=49.355, p<0.001$; group: $\mathrm{F}_{3,15}=58.024, p<0.001$; interaction: $\mathrm{F}_{6,30}=15.624$, $p<0.001$ ). ${ }^{* * *} p<0.001$ vs. sham group; ${ }^{\#} p<0.05$ or ${ }^{\# \#} p<0.001$ vs susceptible+saline group. (D) SPT (time: $\mathrm{F}_{1,5}=1.808, p=0.271 ;$ group: $\mathrm{F}_{3,15}=$ 35.500, $p<0.001$; interaction: $\left.\mathrm{F}_{3,15}=18.718, p<0.001\right) .{ }^{* *} p<0.01$ or ${ }^{* * *} p<0.001$. Data are shown as mean \pm standard error of mean $(\mathrm{n}=6)$.

NS, not significant.

vation and a 24-hour exposure to two identical bottles, one is water, and another is $1 \%$ sucrose solution. The bottles containing water and sucrose were weighed before and at the end of this period and the sucrose preference was determined. ${ }^{34)}$

\section{Enzyme-linked Immunosorbent Assay (ELISA)}

The rat IL-6, IL-10, IL-1 $\beta$ and TNF- $\alpha$ ELISA kits were purchased from MDL Biotech (Beijing, China; $n=7$ or 10 for each group). The serum samples were prepared from whole heart blood samples after centrifugation at $3,000 \times g$ for 10 minutes. The mPFC and spinal cord tissues were homogenized with saline and the homogenate was centrifuged for 10 minutes at $2,500 \mathrm{rpm}$ at $4^{\circ} \mathrm{C}$ in order to ob- tain the supernatant. The $10-\mu \mathrm{l}$ samples and $60-\mu$ l dilution buffer were added to the wells followed by incubation at room temperature for 60 minutes. Then washing the plates and adding zymolytes into the wells, the absorbance was measured on a spectrophotometer at $450 \mathrm{~nm}$. The concentrations were calculated to the amount of standard protein of each sample.

\section{Statistical Analyses}

The data show as the mean \pm standard error of the mean. Analysis was performed using IBM SPSS Statistics software, ver. 20 (IBM Corp., Armonk, NY, USA). Comparisons between groups were performed using the one-way analysis of variance (ANOVA) or two-way 
ANOVA, followed by post-hoc Tukey test. Hierarchical cluster analysis of SPT was applied to classify the anhedonia susceptible or unsusceptible rats. The $p$ values of less than 0.05 were considered statistically significant.

\section{RESULTS}

\section{Comparison of MWT and SPT between Anhedonia Susceptible and Unsusceptible Rats}

SNI is a classical animal model of CNP involving a lesion of terminal branches of the sciatic nerve. ${ }^{35)}$ Interestingly, SNI significantly decreased sucrose preference on day 14 and 21, but not on day 7 after modeling (data not shown). Anhedonia susceptible and unsusceptible rats were further divided by hierarchical cluster analysis of SPT (Fig.
1B). Withdraw threshold was significantly decreased in both anhedonia susceptible and unsusceptible rats as compared with that of control on day 7, 14 and 21 individually after SNI surgery. Between anhedonia susceptible and unsusceptible rats, however, there are no any statistical differences (Fig. 1C). On day 7 after modeling, there are no significant differences among the three groups. Intriguingly, anhedonia susceptible rats significantly decreased sucrose preference as compared with those in the sham and unsusceptible rats on day 14 and 21 after SNI modeling (Fig. 1D). These findings suggest that SNI model is capable to elicit the emergence of anhedonia.
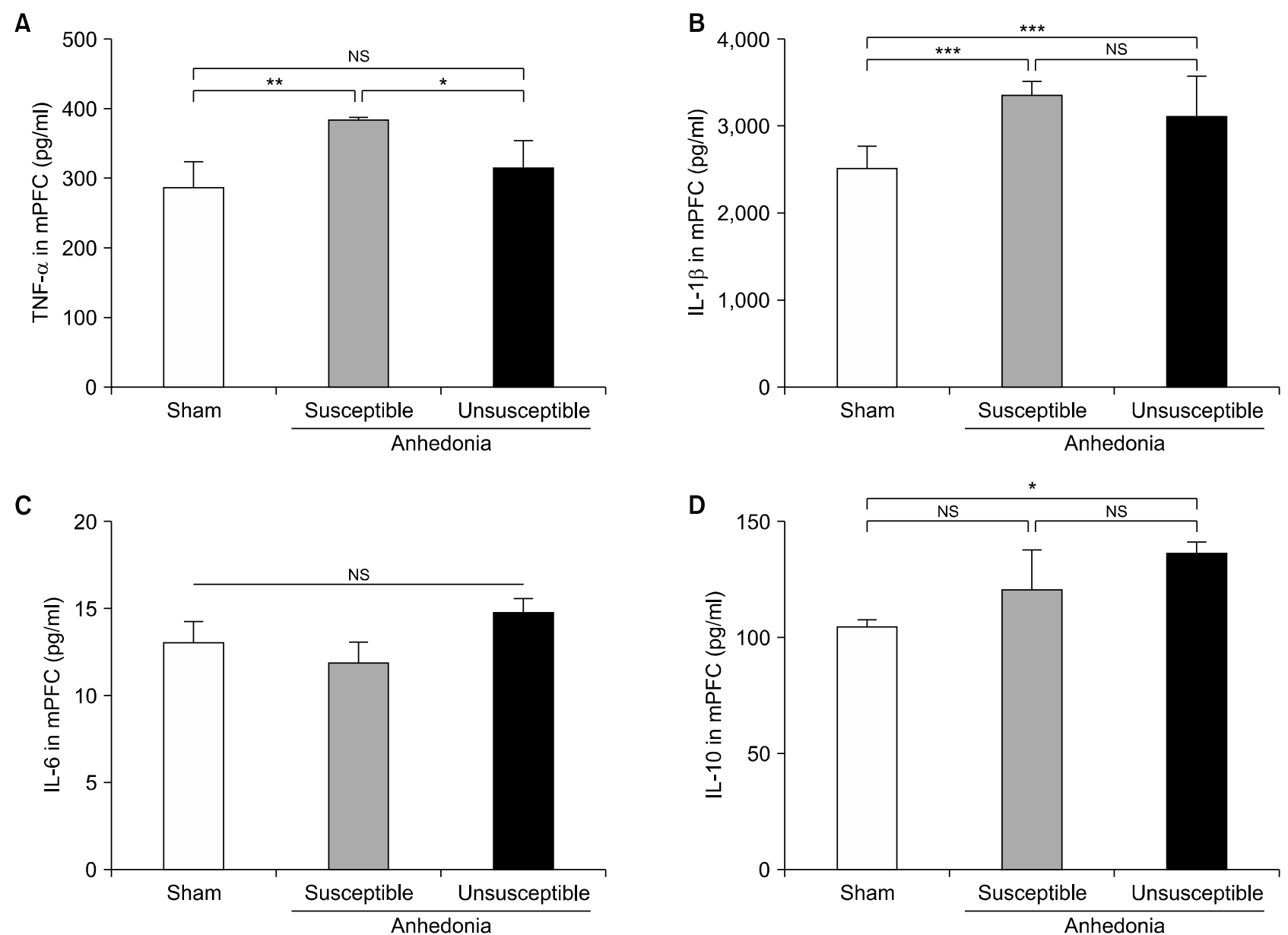

Fig. 3. Expressions of inflammatory cytokines in the medial prefrontal cortex (mPFC). (A) Tumor necrosis factor (TNF)- $\alpha$ level in the mPFC $\left(\mathrm{F}_{2,15}=\right.$ 6.045, $p=0.012)$. (B) Interleukin (IL)-1 $\beta$ level in the mPFC $\left(F_{2,15}=17.222, p<0.001\right)$. (C) IL-6 level in the $\mathrm{mPFC}\left(\mathrm{F}_{2,15}=1.365, p=0.285\right)$. (D) IL-10 level in the $\operatorname{mPFC}\left(\mathrm{F}_{2,15}=5.498, p=0.016\right)$. Data are shown as mean \pm standard error of mean $(\mathrm{n}=6)$.

NS, not significant.

${ }^{*} p<0.05,{ }^{* *} p<0.01$, or ${ }^{* * *} p<0.01$. 


\section{Differential Levels of Inflammatory Cytokines in the mPFC of Anhedonia Susceptible and Unsusceptible Rats}

One-way ANOVA was applied to evaluate the differential levels of TNF- $\alpha$, IL-1 $\beta$, IL- 6 , and IL-10 in the mPFC of rats. Anhedonia susceptible rats demonstrated that TNF- $\alpha$ was significantly up-regulated as compared with that of sham and unsusceptible rats (Fig. 2A). Compared with sham group, rats in anhedonia susceptible and unsusceptible group significantly increased the levels of IL-1 $\beta$. In contrast, there are no any differences in the levels of IL-1 $\beta$ between the anhedoania susceptible and unsusceptible rats (Fig. 2B). Interestingly, IL-6, a pro-inflammatory cytokine, fails to show statistical differences among the three groups (Fig. 2C). Furthermore, compar-
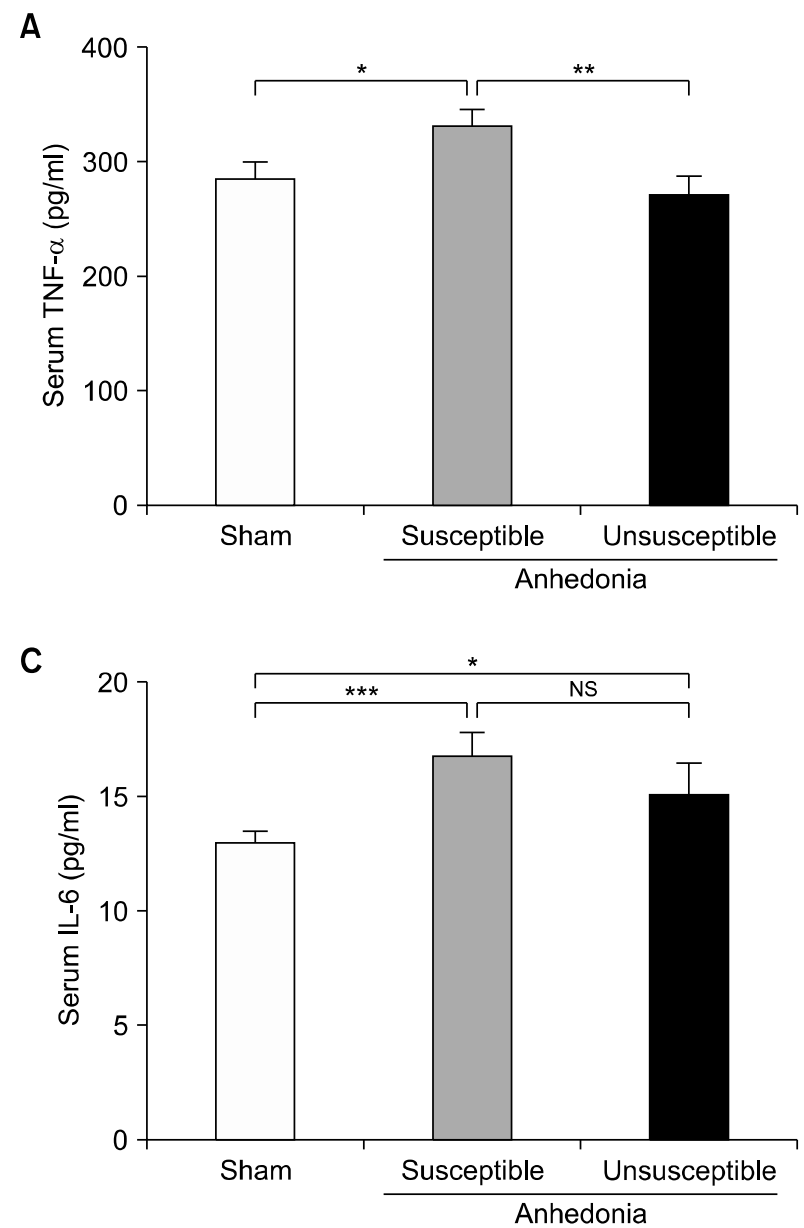

ison of IL-10 level between sham and unsusceptible rats suggested a statistical difference. Although there is a slighter increase of IL-10 level in the anhedonia susceptible rats, post-hoc using Tukey showed no statistical differences. Finally, we showed that IL-10 level was not altered in susceptible as compared with those in the unsusceptible group (Fig. 2D).

\section{Serum Levels of Inflammatory Cytokines in Anhedonia Susceptible and Unsusceptible Rats}

Serum level of TNF- $\alpha$ was significantly increased in the anhedonia susceptible rats as individually compared with those of sham and unsusceptible (Fig. 3A). In addition to IL-1 $\beta$, anhedonia susceptible and unsusceptible were individually increased in relative to those rats in the sham
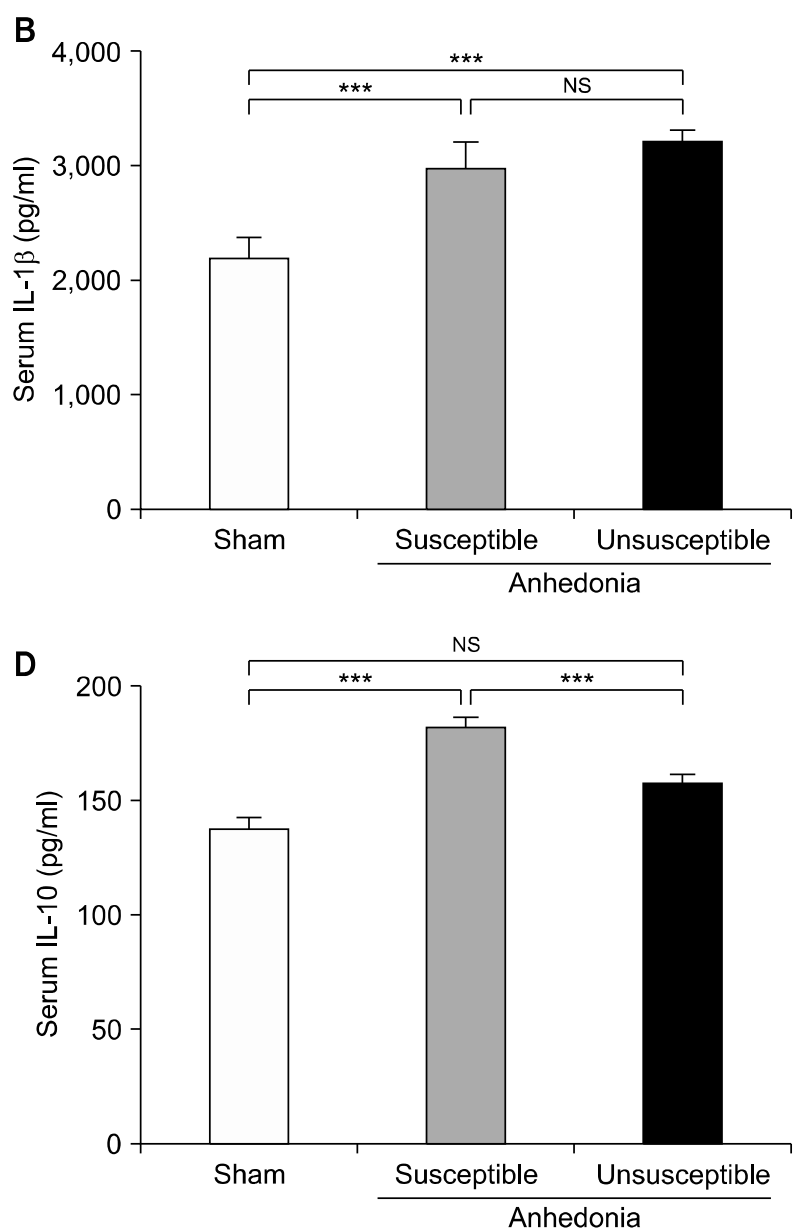

Fig. 4. Expressions of inflammatory cytokines in the serum. (A) Tumor necrosis factor $(\mathrm{TNF})-\alpha$ level in the serum $\left(\mathrm{F}_{2,15}=4.401, p=0.031\right)$. $(\mathrm{B})$ Interleukin (IL)-1 $\beta$ level in the serum $\left(F_{2,15}=147.506, p<0.001\right)$. $(C)$ IL-6 level in the serum $\left(F_{2,15}=7.352, p=0.006\right)$. $(D)$ IL-10 level in the serum $\left(F_{2,15}=\right.$ $64.295, p<0.001)$. Data are shown as mean \pm standard error of mean $(n=6)$.

NS, not significant.

${ }^{*} p<0.05,{ }^{* *} p<0.01$, or ${ }^{* * *} p<0.01$. 
group (Fig. 3B). IL-6 was significantly up-regulated in the anhedonia susceptible rats as compared with that of sham rats (Fig. 3C). Moreover, compared with sham group, anhedonia susceptible group, but not unsusceptible group, significantly increased the serum level of IL-10. Additionally, IL-10 was significantly decreased in the anhedonia unsusceptible vs. the susceptible rats (Fig. 3D).

\section{Expression of Inflammatory Cytokines in the L2-5 Spinal Cord}

The pathogenic mechanisms underlying CNP are highly related to the dysfunction of spinal cord. ${ }^{36)}$ Here we showed that anhedonia susceptible rats markedly increased the spinal TNF- $\alpha$ level. Anhedonia unsusceptible rats furtherly up-regulated the TNF- $\alpha$ in the intumescentia lumbalis of spinal cord (Fig. 4A). Both anhedonia susceptible and unsusceptible showed an increase of IL- $\beta$ as compared with that of sham group, respectively (Fig. 4B). In addition to IL-1 $\beta$, anhedonia susceptible and unsusceptible rats significantly increased IL-6 in relative to rats in the sham group (Fig. 4C). However, spinal level of IL-10 did not show any statistical differences among the three groups (Fig. 4D).

\section{Effects of Ketamine and Parecoxib on MWT and SPT in SNI-induced Anhedonia Susceptible Rats}

Fast-acting antidepressant effects of ketamine have attracted more attentions. ${ }^{37,38)}$ In the present study, 46 rats were enrolled to mimic CNP model via SNI surgery. After hierarchical cluster analysis of SPT, 18 anhedonia suscep-
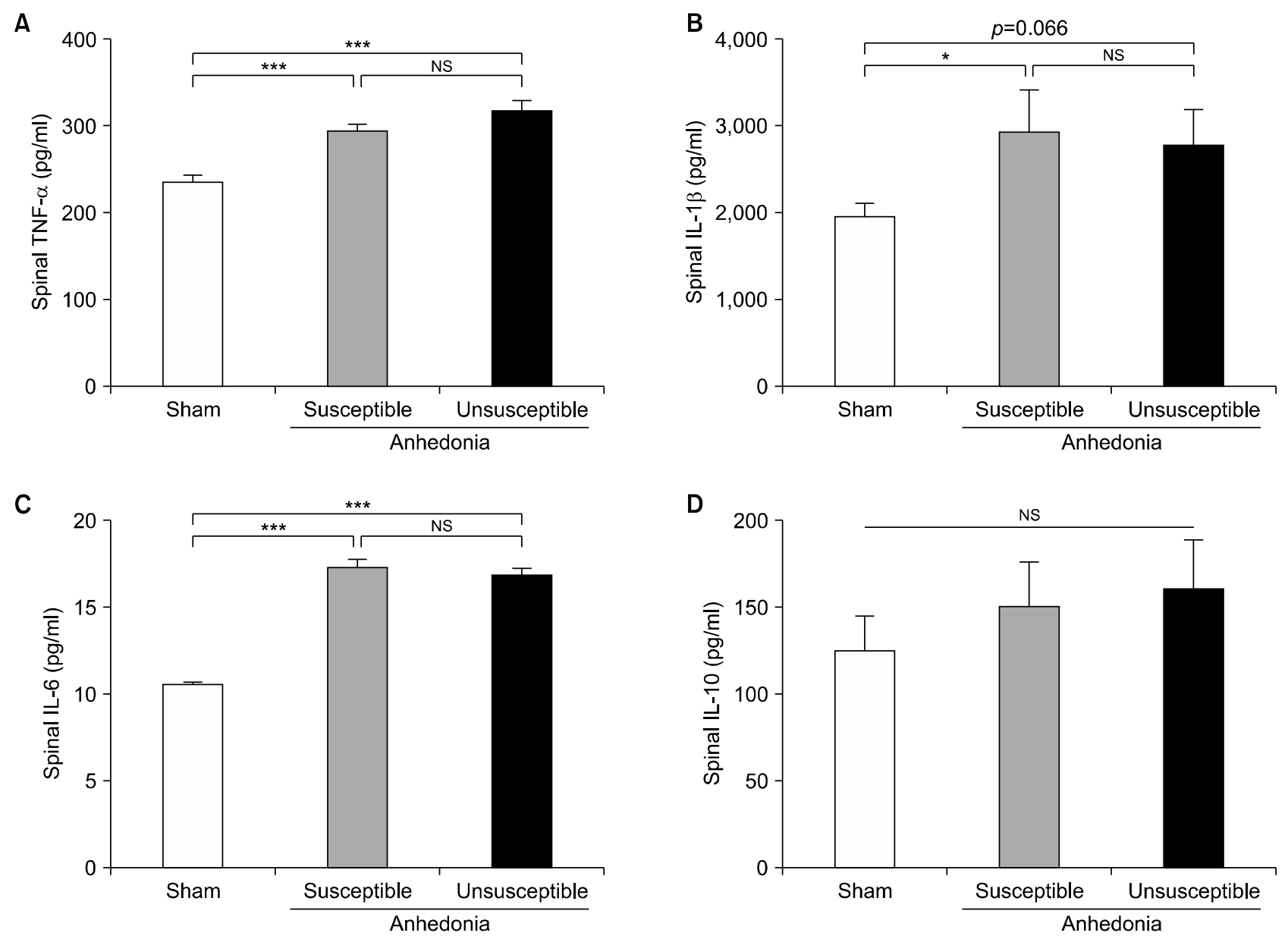

Fig. 5. Expressions of inflammatory cytokines in the L2-5 spinal cord. (A) Tumor necrosis factor (TNF)- $\alpha$ level in the L2-5 spinal cord ( $\mathrm{F}_{2,15}=29.786$, $p<0.001)$. (B) Interleukin (IL)-1 $\beta$ level in the L2-5 spinal cord $\left(\mathrm{F}_{2,15}=4.944, p=0.022\right)$. (C) IL-6 level in the L2-5 spinal cord $\left(\mathrm{F}_{2,15}=175.297, p<\right.$ 0.001). (D) IL-10 level in the L2-5 spinal cord $\left(\mathrm{F}_{2,15}=2.025, p=0.167\right)$. Data are shown as mean \pm standard error of mean ( $\left.\mathrm{n}=6\right)$.

NS, not significant.

${ }^{*} p<0.05,{ }^{* * *} p<0.01$. 
tible rats were selected for further study. Anhedonia susceptible rats (Fig. 5B) were treated with a single dose of ketamie $(10 \mathrm{mg} / \mathrm{kg})$, and that ketamine significantly restored SNI-induced decrease in scores of MWT and SPT (Fig. 5C, 5D). Additionally, paracoxib is a commonly-used analgesic, and its pharmacological property is mainly through inhibiting COX2 receptor to exert an anti-inflammatory action. Interestingly, paracoxib improved the decreased $\mathrm{MWT}$, but failed to show favorable effects on SPT (Fig. 5C, 5D). These findings suggest that ketamine has improving effects on both pain and anhedonia, while paracoxib only alleviates pain symptoms.

\section{DISCUSSION}

$\mathrm{SNI}$, an easily operated animal model of CNP, has been commonly evaluated by examining its mechanical hyperalgesia. ${ }^{39)}$ Several lines of evidence indicate that $\mathrm{SNI}$ is characterized by its earlier-onset and longer-lasting symptoms for better investigating the CNP. ${ }^{39,40)}$ In the present study, we observed that SNI rats significantly decreased the withdraw threshold and sucrose preference than those in control rats, supporting the fact that chronic pain has potential to elicit the emergence of the core symptom of depression-anhedonia. It is commonly recognized that chronic pain and depression might mutually exacerbate one another, finally leading to a poor prognosis.,41) Although there are a large number of studies focusing on the comorbidity of depression and pain, ${ }^{42,43)}$ there is no study investigating the role of chronic pain, particularly $\mathrm{CNP}$, in the core symptoms of depression-anhedoania. Furthermore, we divided the SNI rats into anhedonia susceptible and unsusceptible by hierarchical cluster analysis of SPT. Most importantly, our statistical data demonstrated that approximately $40 \%$ of SNI rats were susceptible to anhedonia. Our group and others, however, suggested that approximately $70 \%$ of mice are susceptible to depression in an animal model of chronic social defeat stress. ${ }^{15,18,44)}$ We think the major reason for this difference is that the diagnostic standards for anhedonia are more stringent than that of depression. In addition, distinct animal models and strains would be contributing factors for the discrepancy of incidence.

The literature on the mPFC is mainly dominated by a large number of studies on its physiological role in mood disorder. ${ }^{45,46)}$ However, the mPFC also plays a key role in the pathogenesis and therapeutic mechanisms of chronic pain. ${ }^{47,48)}$ Similarly, other studies implicate that deactivation of mPFC impairs neural circuit of rewarding effects. ${ }^{49,50)}$ It is therefore likely that mPFC lesions would cause psychological damage to the hedonia because of abnormality in the rewarding systems. ${ }^{51)}$ There is evidence that $\mathrm{mPFC}$ lesions are potentially related to the over-activation of inflammatory response in this brain region. ${ }^{52)}$ In this study, we found that abnormally activated pro-inflammatory cytokines TNF- $\alpha$ and IL-1 $\beta$, but not IL-6, in the mPFC of brain, might contribute to anhedonia susceptibility vs. unsusceptibility. We previously demonstrated that IL-6 in the peripheral serum, but not in the mPFC, may confer susceptible to stress in a rat model of $\mathrm{LH}^{53)}$ consistent with the findings of the present study. Interestingly, also in the mPFC, anti-inflammatory cytokine IL-10 may protect against anhedonia susceptibility. However, the precise mechanisms underlying the role of brain level of IL-10 in the anhedonia unsusceptibility remain unknown.

Disorders in the peripheral levels of inflammatory cytokines might lead to the development of depression. ${ }^{20,21)}$ Immune cells peripherally produce and release pro-inflammatory cytokines, finally impacting the brain to cause an aggravation of sickness behavior and the development of depression in vulnerable individuals. ${ }^{54)}$ Peripheral inflammatory response is therefore a key pathogenic trigger that might increase the risk of depression. ${ }^{54)}$ As described above, we previously reported that peripheral level of serum IL-6 is an aggravating factor for stress susceptibility in a rodent model of $\mathrm{LH}^{53)}$ Intriguingly, in the present study, our results showed that not only IL-6, but also other inflammatory cytokines including IL-1 $\beta$, IL- 6 and IL-10 in the serum, would promote the CNP-related anhedonia susceptibility. However, so far, there are few studies focusing on the role of serum inflammatory response in the pain-related anhedonia. Dellarole et al. ${ }^{29)}$ displayed that neuropathic pain-induced depressive-like symptoms are dependent on TNF signaling through its receptor TNFR1. Further detailed studies on the role peripheral inflammation in pain-related depression or anhedonia are required.

Spinal cord is of great importance to the production and conduction of pain sensation. ${ }^{55)}$ It is well believed that spinal cord stimulation is helpful to alleviate chronic pain. ${ }^{56,57)}$ Additionally, many studies indicated patients with traumatic spinal cord injury are prone to easily suffer 
from depression. ${ }^{58)}$ However, the precise mechanisms underlying the crosstalk between the spinal cord and brain are elusive. In the present study, we failed to find the statistical differences in the inflammatory cytokines between anhedonia susceptibility and unsusceptibility in a SNI rat model of CNP. But SNI rats including anhedonia susceptible and unsusceptible rats significantly increased the spinal levels of TNF- $\alpha$, IL-1 $\beta$ and IL- 6 , consistent with studies showing over-activated microglia-derived inflammatory cytokines greatly promote the development of CNP.

Inflammatory responses are complex in the pathogenesis and therapeutic mechanisms of pain and depression, which are more complicated in the comorbidity of pain and depression. Xie et al. ${ }^{34)}$ demonstrated that IL-1 $\beta$, IL-6, TNF- $\alpha$, and IL-10 in PFC were significantly increased in depression rats, whereas these inflammatory cytokines were significantly decreased in non-depression rats as compared with that of depression rats. However, our results demonstrated that IL-6 was not altered, and that the levels of IL-1 $\beta$ and IL-10 were not lowered in mPFC of anhedonia unsusceptible rats. The discrepancy seems to be connected with different commercially ELISA kits, different study protocol or different time-point of tissue collection.

Ketamine, an antagonist of $\mathrm{N}$-methyl-D-aspartic acid receptor, has been reported to have a fast-acting and long-term antidepressant effect, especially for refractory depression. ${ }^{38,59,60)}$ In this study, we observed that ketamine is effective in alleviation of anhedonia and pain symptoms. We previously reported that regulation of inflammatory response might be involved in the antidepressant effects of ketamine, indirectly supporting the possibility that inhibiting abnormally activated inflammatory response might, at least partially, contribute to the therapeutic actions of ketamine on depression. ${ }^{61)}$ Interestingly, parecoxib, an-anti-inflammatory agent commonly applied to treat pain, ${ }^{62)}$ but failed to alleviate anhedonia symptoms. Although the reasons underlying the discrepancy between the therapeutic effects of ketamine and parecoxib on anhedonia are currently unknown, we hypothesized that differential pharmacological properties of the drugs are a main cause. Future studies on the role of inflammatory response in the therapeutic mechanisms of ketamine for pain-related depression are greatly needed.

In conclusion, this study suggests that inflammatory cytokines in the mPFC and serum might contribute to anhe- donia susceptibility vs. unsusceptibility in a rat model of SNI. Monoclonal antibodies targeting IL- 6 and TNF- $\alpha$ would provide an available strategy for treatment of pain and/or depression since these biological agents have been clinically used for rheumatoid arthritis treatment. Furthermore, ketamine is effective in alleviating anhedonia symptoms in a rat model of SNI. Further clinical studies on the effects of ketamine on pain-related depression or the comorbidity of pain and depression are required.

\section{- Acknowledgments}

This study was supported by grants from the National Natural Science Foundation of China (No.: 81771159, 81500931 and 81703482) and "333 Project" of Jiangsu Province (No.: BRA2016122).

\section{REFERENCES}

1. Gerrits MM, van Marwijk HW, van Oppen $P$, van der Horst $H$, Penninx BW. Longitudinal association between pain, and depression and anxiety over four years. J Psychosom Res 2015; 78:64-70.

2. Goesling J, Clauw DJ, Hassett AL. Pain and depression: an integrative review of neurobiological and psychological factors. Curr Psychiatry Rep 2013;15:421.

3. Bair MJ, Robinson RL, Katon W, Kroenke K. Depression and pain comorbidity: a literature review. Arch Intern Med 2003; 163:2433-2445.

4. de Leon-Casasola OA. Opioids for chronic pain: new evidence, new strategies, safe prescribing. Am JMed 2013;126(3 Supp/ 1):S3-S11.

5. Nalamachu S. An overview of pain management: the clinical efficacy and value of treatment. Am JManag Care 2013;19(14 Suppl):s261-s266.

6. Finnerup NB, Attal N, Haroutounian S, McNicol E, Baron R, Dworkin RH, et al. Pharmacotherapy for neuropathic pain in adults: a systematic review and meta-analysis. Lancet Neurol 2015;14:162-173.

7. Dworkin RH, O'Connor AB, Audette J, Baron R, Gourlay GK, Haanpää $\mathrm{ML}$, et al. Recommendations for the pharmacological management of neuropathic pain: an overview and literature update. Mayo Clin Proc 2010;85(3 Suppl):S3-S14.

8. Gilmartin JF, Väätäinen $S$, Törmälehto $S$, Bell JS, Lönnroos $E$, Salo L, et al. Depressive symptoms are associated with analgesic use in people with Alzheimer's disease: Kuopio ALSOVA study. PLoS One 2015;10:e0117926.

9. Mohr P, Bitter I, Svestka J, Seifritz E, Karamustafalioglu O, Koponen $\mathrm{H}$, et al. Management of depression in the presence of pain symptoms. Psychiatr Danub 2010;22:4-13.

10. Winer ES, Nadorff MR, Ellis TE, Allen JG, Herrera S, Salem T. Anhedonia predicts suicidal ideation in a large psychiatric inpatient sample. Psychiatry Res 2014;218:124-128. 
11. Rizvi SJ, Quilty LC, Sproule BA, Cyriac A, Michael Bagby R, Kennedy SH. Development and validation of the Dimensional Anhedonia Rating Scale (DARS) in a community sample and individuals with major depression. Psychiatry Res 2015;229: 109-119.

12. Der-Avakian A, Markou A. The neurobiology of anhedonia and other reward-related deficits. Trends Neurosci 2012;35. 68-77.

13. Page GG, Opp MR, Kozachik SL. Sex differences in sleep, anhedonia, and HPA axis activity in a rat model of chronic social defeat. Neurobio/ Stress 2016;3:105-113.

14. Yamamoto H, Kamegaya E, Hagino Y, Takamatsu Y, Sawada W, Matsuzawa M, et al. Loss of GluN2D subunit results in social recognition deficit, social stress, 5-HT2C receptor dysfunction, and anhedonia in mice. Neuropharmacology 2017; 112:188-197.

15. Yang C, Ren Q, Qu Y, Zhang JC, Ma M, Dong C, et al. Mechanistic target of rapamycin-independent antidepressant effects of (R)-Ketamine in a social defeat stress model. Biol Psychiatry 2018;83:18-28.

16. Yang C, Shirayama Y, Zhang JC, Ren Q, Yao W, Ma M, et al. $R$-ketamine: a rapid-onset and sustained antidepressant without psychotomimetic side effects. Transl Psychiatry 2015;5: e632.

17. Riga D, Theijs JT, De Vries TJ, Smit AB, Spijker S. Social defeat-induced anhedonia: effects on operant sucrose-seeking behavior. Front Behav Neurosci 2015:9:195.

18. Yang C, Fujita Y, Ren Q, Ma M, Dong C, Hashimoto K. Bifidobacterium in the gut microbiota confer resilience to chronic social defeat stress in mice. Sci Rep 2017;7:45942.

19. Yang C, Qu Y, Abe M, Nozawa D, Chaki S, Hashimoto K. (R)-Ketamine shows greater potency and longer lasting antidepressant effects than its metabolite $(2 R, 6 R)$-hydroxynorketamine. Biol Psychiatry 2017;82:e43-e44.

20. Ma M, Ren Q, Zhang JC, Hashimoto K. Effects of brilliant blue Gon serum tumor necrosis factor- $\alpha$ levels and depression-like behavior in mice after lipopolysaccharide administration. Clin Psychopharmacol Neurosci 2014;12:31-36.

21. Dean OM, Maes M, Ashton M, Berk L, Kanchanatawan B, Sughondhabirom A, et al. Protocol and rationale-the efficacy of minocycline as an adjunctive treatment for major depressive disorder: a double blind, randomised, placebo controlled trial. Clin Psychopharmacol Neurosci 2014;12:180-188.

22. Dowlati Y, Herrmann N, Swardfager W, Liu H, Sham L, Reim EK, et al. A meta-analysis of cytokines in major depression. Biol Psychiatry 2010;67:446-457.

23. Udina M, Moreno-España J, Capuron L, Navinés R, Farré M, Vieta $\mathrm{E}$, et al. Cytokine-induced depression: current status and novel targets for depression therapy. CNS Neurol Disord Drug Targets 2014;13:1066-1074.

24. Kohler O, Krogh J, Mors O, Benros ME. Inflammation in depression and the potential for anti-inflammatory treatment. Curr Neuropharmacol 2016;14:732-742.
25. Lawson MA, McCusker RH, Kelley KW. Interleukin-1 beta converting enzyme is necessary for development of depression-like behavior following intracerebroventricular administration of lipopolysaccharide to mice. J Neuroinflammation 2013;10:54.

26. Berghen N, Teuwen LA, Westhovens R, Verschueren P. Malignancies and anti-TNF therapy in rheumatoid arthritis: a single-center observational cohort study. Clin Rheumatol 2015;34:1687-1695.

27. Abbott R, Whear R, Nikolaou V, Bethel A, Coon JT, Stein K, et al. Tumour necrosis factor- $\alpha$ inhibitor therapy in chronic physical illness: A systematic review and meta-analysis of the effect on depression and anxiety. J Psychosom Res 2015;79: 175-184.

28. Demirci S, Aynal 1 A, Demirci K, Demirci S, Arıdoğan BC. The serum levels of resistin and its relationship with other proinflammatory cytokines in patients with Alzheimer's disease. Clin Psychopharmacol Neurosci 2017;15:59-63.

29. Dellarole A, Morton P, Brambilla R, Walters W, Summers S, Bernardes D, et al. Neuropathic pain-induced depressive-like behavior and hippocampal neurogenesis and plasticity are dependent on TNFR1 signaling. Brain Behav Immun 2014; 41:65-81.

30. Lopresti AL, Maker GL, Hood SD, Drummond PD. A review of peripheral biomarkers in major depression: the potential of inflammatory and oxidative stress biomarkers. Prog Neuropsychopharmacol Biol Psychiatry 2014;48:102-111.

31. Brunoni AR, Machado-Vieira R, Zarate CA, Valiengo L, Vieira $\mathrm{EL}$, Benseñor IM, et al. Cytokines plasma levels during antidepressant treatment with sertraline and transcranial direct current stimulation (tDCS): results from a factorial, randomized, controlled trial. Psychopharmacology (Berl) 2014;231: 1315-1323.

32. Decosterd I, Woolf CJ. Spared nerve injury: an animal model of persistent peripheral neuropathic pain. Pain 2000;87: 149-158.

33. Song Z, Xiong B, Zheng $\mathrm{H}$, Manyande A, Guan X, Cao F, et al. STAT1 as a downstream mediator of ERK signaling contributes to bone cancer pain by regulating MHC I/ expression in spinal microglia. Brain Behav Immun 2017;60:161-173.

34. Xie ZM, Wang XM, Xu N, Wang J, Pan W, Tang XH, et al. Alterations in the inflammatory cytokines and brain-derived neurotrophic factor contribute to depression-like phenotype after spared nerve injury: improvement by ketamine. Sci Rep 2017;7:3124.

35. Challa SR. Surgical animal models of neuropathic pain: pros and cons. Int I Neurosci 2015;125:170-174.

36. Schmidtko A. Nitric oxide-mediated pain processing in the spinal cord. Handb Exp Pharmacol 2015;227:103-117.

37. Hashimoto K. A BDNF Val66Met polymorphism and ketamine-induced rapid antidepressant action. Clin Psychopharmacol Neurosci 2012;10:59-60.

38. Li SX, Zhang JC, Wu J, Hashimoto K. Antidepressant effects of 
ketamine on depression-like behavior in juvenile mice after neonatal dexamethasone exposure. Clin Psychopharmacol Neurosci 2014;12:124-127.

39. Richner M, Bjerrum OJ, Nykjaer A, Vaegter CB. The spared nerve injury (SNI) model of induced mechanical allodynia in mice. J Vis Exp 2011;(54):3092.

40. Pertin M, Gosselin RD, Decosterd I. The spared nerve injury model of neuropathic pain. Methods Mol Biol 2012;851:205212.

41. Walker AK, Kavelaars A, Heijnen CJ, Dantzer R. Neuroinflammation and comorbidity of pain and depression. Pharmacol Rev 2013;66:80-101.

42. Ashrafioun L, Bishop TM, Conner KR, Pigeon WR. Frequency of prescription opioid misuse and suicidal ideation, planning, and attempts. J Psychiatr Res 2017;92:1-7.

43. Paksarian D, Cui L, Angst J, Ajdacic-Gross V, Rössler W, Merikangas KR. Stability and change in reported age of onset of depression, back pain, and smoking over 29 years in a prospective cohort study. J Psychiatr Res 2017;88:105-112.

44. Ren Q, Ma M, Ishima T, Morisseau C, Yang J, Wagner KM, et al. Gene deficiency and pharmacological inhibition of soluble epoxide hydrolase confers resilience to repeated social defeat stress. Proc Natl Acad Sci U S A 2016; 113:E1944E1952.

45. Han M, Zhang JC, Hashimoto K. Increased levels of C1q in the prefrontal cortex of adult offspring after maternal immune activation: prevention by 7,8-dihydroxyflavone. Clin Psychopharmacol Neurosci 2017;15:64-67.

46. Abelaira HM, Réus GZ, Ignácio ZM, Dos Santos MA, de Moura $A B$, Matos $D$, et al. Effects of ketamine administration on $m T O R$ and reticulum stress signaling pathways in the brain after the infusion of rapamycin into prefrontal cortex. J Psychiatr Res 2017;87:81-87.

47. Guida F, Luongo L, Marmo F, Romano R, lannotta M, Napolitano F, et al. Palmitoylethanolamide reduces pain-related behaviors and restores g/utamatergic synapses homeostasis in the medial prefrontal cortex of neuropathic mice. Mol Brain 2015;8:47.

48. Bräscher AK, Becker S, Hoeppli ME, Schweinhardt P. Different brain circuitries mediating controllable and uncontrollable pain. J Neurosci 2016;36:5013-5025.

49. Liu Z, Wang Y, Cai L, Li Y, Chen B, Dong Y, et al. Prefrontal cortex to accumbens projections in sleep regulation of reward. J Neurosci 2016;36:7897-7910.
50. Petykó Z, Gálosi R, Tóth A, Máté K, Szabó I, Szabó I, et al. Responses of rat medial prefrontal cortical neurons to Pavlovian conditioned stimuli and to delivery of appetitive reward. Behav Brain Res 2015;287:109-119.

51. Ferenczi EA, Zalocusky KA, Liston C, Grosenick L, Warden MR, Amatya D, et al. Prefrontal cortical regulation of brainwide circuit dynamics and reward-related behavior. Science 2016;351:aac9698.

52. Sekio M, Seki K. Lipopolysaccharide-induced depressive-like behavior is associated with $\alpha_{1}$-adrenoceptor dependent downregulation of the membrane GluR1 subunit in the mouse medial prefrontal cortex and ventral tegmental area. Int J Neuropsychopharmacol 2014;18:pyu005.

53. Yang C, Shirayama Y, Zhang JC, Ren Q, Hashimoto K. Peripheral interleukin-6 promotes resilience versus susceptibility to inescapable electric stress. Acta Neuropsychiatr 2015;27:312-316.

54. D'Mello C, Swain MG. Immune-to-brain communication pathways in inflammation-associated sickness and depression. Curr Top Behav Neurosci 2017;31:73-94.

55. Guo D, Hu J. Spinal presynaptic inhibition in pain control. Neuroscience 2014;283:95-106.

56. Gjesdal K, Furnes B, Dysvik E. Experiences with spinal cord stimulator in patients with chronic neuropathic back pain. Pain Manag Nurs 2014;15:e13-e24.

57. Baranidharan G, Simpson KH, Dhandapani K. Spinal cord stimulation for visceral pain--a novel approach. Neuromodulation 2014;17:753-758; discussion 758.

58. Siddall PJ, Middleton JW. Spinal cord injury-induced pain: mechanisms and treatments. Pain Manag 2015;5:493-507.

59. Hashimoto K. The R-stereoisomer of ketamine as an alternative for ketamine for treatment-resistant major depression. Clin Psychopharmacol Neurosci 2014;12:72-73.

60. Dang YH, Ma XC, Zhang JC, Ren Q, Wu J, Gao CG, et al. Targeting of NMDA receptors in the treatment of major depression. Curr Pharm Des 2014;20:5151-5159.

61. Yang C, Hong T, Shen J, Ding J, Dai XW, Zhou ZQ, et al. Ketamine exerts antidepressant effects and reduces $I L-1 \beta$ and IL-6 levels in rat prefrontal cortex and hippocampus. Exp Ther Med 2013;5:1093-1096.

62. Gajraj NM. COX-2 inhibitors celecoxib and parecoxib: valuable options for postoperative pain management. Curr Top Med Chem 2007;7:235-249. 